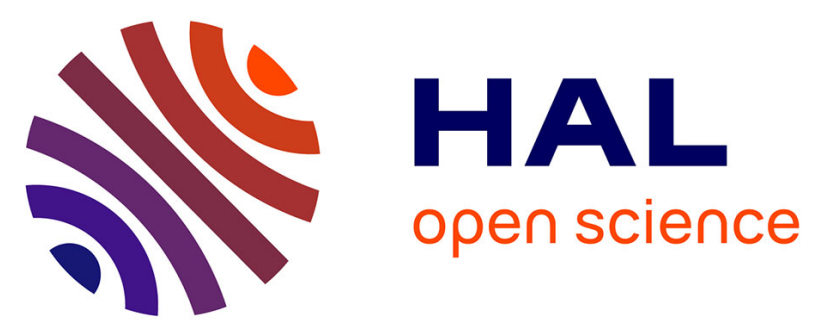

\title{
A Study of Solar Orbiter Spacecraft-Plasma Interactions Effects on Electric Field and Particle Measurements
} Stanislas Guillemant, Milan Maksimovic, Alain Hilgers, Filippo Pantellini, Lamy Laurent, Philippe Louarn, Vincent Génot, Christopher L. Owens, Jean-Charles Mateo-Velez, Pierre Sarrailh, et al.

\section{To cite this version:}

Stanislas Guillemant, Milan Maksimovic, Alain Hilgers, Filippo Pantellini, Lamy Laurent, et al.. A Study of Solar Orbiter Spacecraft-Plasma Interactions Effects on Electric Field and Particle Measurements. IEEE Transactions on Plasma Science, 2017, 45 (9), page 2578-2587. 10.1109/TPS.2017.2731054 . hal-01706575

\section{HAL Id: hal-01706575 \\ https://hal.science/hal-01706575}

Submitted on 12 Feb 2018

HAL is a multi-disciplinary open access archive for the deposit and dissemination of scientific research documents, whether they are published or not. The documents may come from teaching and research institutions in France or abroad, or from public or private research centers.
L'archive ouverte pluridisciplinaire HAL, est destinée au dépôt et à la diffusion de documents scientifiques de niveau recherche, publiés ou non, émanant des établissements d'enseignement et de recherche français ou étrangers, des laboratoires publics ou privés. 


\title{
A STUDY OF SOLAR ORBITER SPACECRAFT PLASMA INTERACTIONS EFFECTS ON ELECTRIC FIELD AND PARTICLE MEASUREMENTS
}

\author{
Guillemant S. ${ }^{(1,2)}$, Maksimovic M. ${ }^{(1)}$, Hilgers A. ${ }^{(3)}$, Pantellini F. ${ }^{(1)}$, Lamy L. ${ }^{(1)}$, Louarn P..$^{(2)}$, Génot V. ${ }^{(2)}$, Owen \\ C.J. ${ }^{(4)}$, Matéo-Vélez J.-C. ${ }^{(5)}$, Sarrailh P. ${ }^{(5)}$, Hess S. ${ }^{(5)}$, Vecchio A. ${ }^{(1)}$ \\ ${ }^{(1)}$ LESIA/CNRS, Observatoire de Paris, Section de Meudon, 5 place Jules Janssen, 92195 MEUDON Cedex, France, \\ Email : milan.maksimovic@obspm.fr \\ ${ }^{(2)}$ IRAP, 9, avenue du Colonel Roche, BP 44346, 31028 Toulouse Cedex 4, France, Email : \\ stanislas.guillemant@irap.omp.eu \\ ${ }^{(3)}$ ESA/ESTEC, Noordwijk, The Netherlands, Email : alain.hilgers@esa.int \\ ${ }^{(4)}$ MSSL/UCL, Dorking, Surrey, United Kingdom,Email: c.owen@ucl.ac.uk \\ ${ }^{(5)}$ ONERA, 2 Avenue Edouard Belin, 31000 Toulouse, France, Email: jean-charles.mateo_velez@onera.fr
}

\begin{abstract}
We present numerical simulations of the Solar Orbiter spacecraft/plasma interaction performed with the Spacecraft Plasma Interaction System (SPIS) software. Solar Orbiter, to be launched in October 2018, is dedicated to observe the Sun and the solar wind. The spacecraft will be equipped with both in-situ and remote sensing instruments and will approach the Sun as close as $0.28 \mathrm{AU}$. The whole spacecraft will be submitted to an intense electromagnetic radiation flux (10 times that at Earth) leading to specific thermal and spacecraft plasma interactions issues. This paper investigates plasma interaction effects with two instruments: the Radio and Plasma Waves (RPW) and the Electron Analyzer System (EAS).
\end{abstract}

\section{INTRODUCTION}

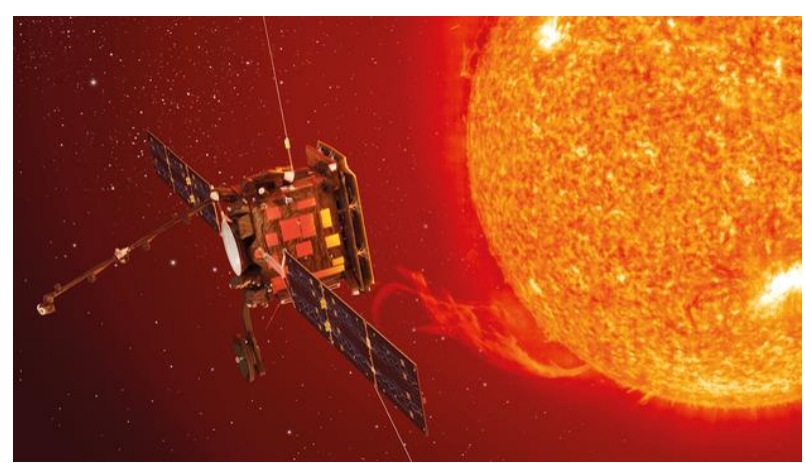

Figure 1. Solar Orbiter near the Sun (credit: ESA)

The Solar Orbiter spacecraft (SC) (see Fig. 1), to be launched in 2018, is the first ESA Medium-class mission from the Cosmic Vision program 2015-2025, with NASA participation. This satellite will orbit the Sun at a distance between 1.4 and 0.28 A.U (with nominal scientific orbit between $\sim 0.8$ and 0.28 A.U.)., observing the star from both within and outside ecliptic regions thanks to an inclination of $25^{\circ}$ during the nominal mission operation and potentially increased to $34^{\circ}$ during the extended phase of the mission. The mission aims at understanding the solar activity effects on the inner heliosphere though in situ and remote observations.

The location of the two instruments of interest for this work onto the spacecraft is indicated on Fig. 2. The Radio and Plasma Waves device (RPW) consists in three conducting antennas of more than $6 \mathrm{~m}$ length measuring the ambient electric and magnetic field fluctuations from DC to several $\mathrm{kHz}$. The potential on each antenna is a function of the local plasma conditions and in particular a function of the plasma's ambient electric field. As a consequence, the component of the ambient electric field coplanar with the antennas can in principle be deduced from the differences between the potentials on the three antennas, provided that the spacecraft induced effects are known.

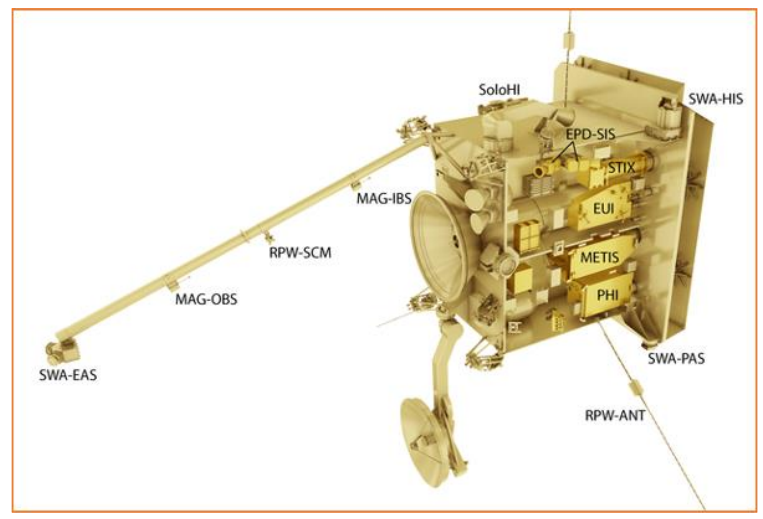

Figure 2. Localisation of Solar Orbiter scientific instruments on the spacecraft (credit: ESA)

The Electron Analyzer System (EAS) is a part of the Solar Wind plasma Analyzer (SWA). EAS measures the electron bulk properties (including density $N$, velocity $V$, and temperature $T$ ) of the solar wind, between 0.28 and $0.8 \mathrm{AU}$ heliocentric distance. It is made of a pair of top-hat electrostatic analyzers with aperture deflection plates mounted in the shadow of the spacecraft at the end of the instrument boom. The two sensors provide an almost full $4 \pi$ sr field of view subject only to minor 
blockage by the spacecraft and its appendages. The sensors measure electron fluxes in the $1 \mathrm{eV}$ to $5 \mathrm{keV}$ energy range with $10-12 \%$ precision and 10 degrees' angular resolution. Moments of the electron velocity distribution at $4 \mathrm{~s}$ time resolution will be routinely returned to ground even though the sensors can sample full 3D distributions at a higher rate and 2D electron pitch angle distributions at $0.125 \mathrm{~s}$ rate during short periods of burst mode.

At Solar Orbiter perihelion $(\sim 0.28$ AU) the solar radiation flux is about 10 times stronger than at $1 \mathrm{AU}$ and the solar wind is also significantly denser and hotter than at 1 AU. A previous study [1] suggested that the EAS detector may be contaminated by important fluxes of low energy secondary and photoelectrons, emitted by the spacecraft itself, and deflected towards the detectors by the local structure of the plasma potential. The local plasma potential is indeed modified by various spacecraft induced effects such as the charging of covering material, the presence of an ion wake and high secondary electron / photoelectron densities, which in turn affect the measured thermal electrons Energy Distribution Functions (EDF). Compared with theoretical undisturbed EDF, simulations show that EAS may measure density excess of more than $130 \%$ at perihelion with the largest discrepancy in the electron flow direction. On the other hand, the three RPW antennas will be surrounded by electron clouds inducing noticeable deformations of the local electrostatic pattern. In addition, the antennas might bend due to material expansion on their sunlit faces as at perihelion antennas temperature may reach $500-600^{\circ} \mathrm{C}$. Finally, Solar Orbiter body elements covered with dielectric materials may sufficiently charge to affect measurements of the ambient low frequency electric field .

Numerical simulations are the only mean to make a realistic and accurate quantitative model of the satellite induced perturbations on instrument measurements. The Spacecraft Plasma Interaction System (SPIS) [2] software is used simulate the Solar Orbiter plasma environment and to evaluate the quantitative impact of the various parts of the spacecraft onto RPW and EAS measurements. The SPIS project aims at developing a software toolkit for spacecraft-plasma interactions and spacecraft charging modeling. Its numerical kernel is a 3D unstructured Particle-In-Cell plasma model (PIC) and a Poisson solver. It is supported by a consortium of users and developers, the Spacecraft Plasma Interaction Network in Europe, which tests and validates the code [3]. Recent SPIS developments have enabled the user to add scientific instruments (Langmuir probes, particle detectors, ...) in the simulation domain as to mimic expected measurements [4].

In the following, SPIS simulations of Solar Orbiter at its perihelion will be presented. In the first part we show simulations where a spatially constant electric field has been added as to mimic the effect of low frequency electric field fluctuations onto the RPW antennas. The aim is to provide estimates of the effective length for the three possible antenna couples, the only way to link the ambient electric field to the antenna potentials. In a second part, spacecraft/plasma interaction impacts on EAS measurements will be discussed. These include the modifications of the velocity moments due to spacecraft charging and particle emission and to the deflection of the incoming particle flow due to the local electrostatic plasma potential structure.

The objective of this work is to provide a better understanding of upcoming on-board measurements and provide estimates of the corrections required to recover the unperturbed local plasma parameters.

\section{SIMULATION CONFIGURATION}

\subsection{Geometry model}

The spacecraft configuration used in our simulations is presented on Fig. 3. It is a simplified model of the satellite made with the Gmsh software, the SPIS embedded CAD modeling tool) as we evidently cannot reproduce in full details the real structure of the spacecraft. The main elements have been modeled in $3 \mathrm{D}$, respecting their real (or sometimes supposed) dimensions as they were at the time of the simulation. Dimensions and covering materials may still change depending on technical and/or industrial constraints. In any case, for this work, all surfaces are electrically conducting except for the two front faces of the solar arrays.

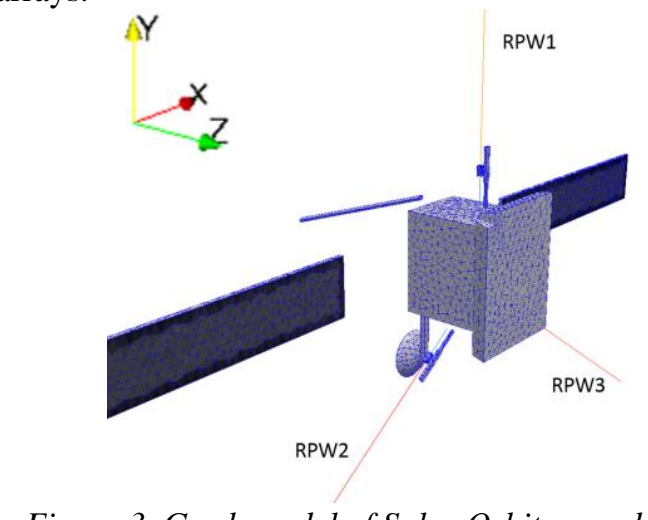

Figure 3. Gmsh model of Solar Orbiter used in SPIS

On Fig. 3, one identifies the main elements: the satellite body, the sunshield, the High Gain Antenna (HGA) dish and its mast, the two solar arrays and the boom at the rear on which outer end the EAS instrument (not yet represented on this model) will be mounted. Also shown are the three RPW $6.5 \mathrm{~m}$ long thin antennas (average radius of $1.3 \mathrm{~cm}$ ) in red and their supporting booms (modeled as thin wires) in blue and three sunshields to protect the preamplifier boxes (in which the stacers are stowed before the antenna deployment). Gmsh produces an unstructured grid of $\sim 340000$ tetrahedra filling an 
ovoid of about $36 \times 36 \times 40 \mathrm{~m}$ containing the spacecraft.

\subsection{Environment and SPIS parameters}

\begin{tabular}{|c|c|}
\hline Environment parameters & $\begin{array}{c}\text { Values at 0.28 AU from } \\
\text { the Sun }\end{array}$ \\
\hline Sun flux (\# 1 AU) & 12.76 \\
\hline$N$ of Electron and $H+\left(\mathrm{m}^{-3}\right)$ & $1.04 \times 10^{8}$ \\
\hline Electron temperature $(\mathrm{eV})$ & 21.37 \\
\hline Proton temperature (eV) & 27 \\
\hline $\begin{array}{c}\text { Spacecraft velocity in X } \\
\text { direction (m/s) }\end{array}$ & 60000.0 \\
\hline $\begin{array}{c}\text { Spacecraft velocity in Z } \\
\text { direction (m/s) }\end{array}$ & 400000.0 \\
\hline $\begin{array}{c}\text { Magnetic field in }-\mathrm{Z} \\
\text { direction }(\mathrm{T})\end{array}$ & $4 \times 10^{-6}$ \\
\hline Debye length (m) & 3.4 \\
\hline
\end{tabular}

Table 1. Environment parameters used in SPIS

We place the study at Solar Orbiter perihelion, i.e. at 0.28 A.U. from the Sun. The plasma parameters used in our simulations are listed in Tab. 1. All species (protons, thermal electrons, secondary electrons and photoelectrons) are computed using a PIC model. The secondary electron emission under electron impact (SEY), as well as the secondary electron emission under proton impact (SEP), are set with a characteristic energy of $2 \mathrm{eV}$ (Maxwellian velocity distribution function). The backscattered electrons are emitted with $2 / 3$ of their initial energy and photoelectrons are emitted with a Maxwellian energy profile with a temperature of $3 \mathrm{eV}$. The Sun is located in the $-Z$ direction, normal to the sunshield. With the SPIS version used here (5.1.9) it is not possible to apply directly an electric field over the simulation box (constant over time and unidirectional). The trick to succeed in finally simulating an $\boldsymbol{E}$ field was to apply magnetic one ( $\boldsymbol{B}$, constant and uniform over the simulation volume), combined with the spacecraft velocity according to its motion along its orbit $(\boldsymbol{V})$, therefore fixing a $\boldsymbol{V} \times \boldsymbol{B}$ induced convection $\boldsymbol{E}$ field. By doing so, we also consider a strong, unrealistic, interplanetary magnetic field in order to clearly see field effects on the RPW antennas, and we also add effects of magnetized particles in the simulation that will be discussed in the next section. In a further work a more realistic environment will be applied. According to the configuration indicated in Table 1, the induced electric field is here set to $E_{Y}=240 \mathrm{mV} / \mathrm{m}$. Note that almost all conducting surfaces are directly connected to the spacecraft ground, meaning that those will have their potential floating at the same value that the Solar Orbiter body. The external surfaces of dielectric elements will charge differentially. However, we apply a capacity between the three RPW antennas and the satellite ground in order to decouple those wires from the body and to derive different potentials on them.

\section{SIMULATION OF RPW ANTENNAS}

\subsection{Simulation results}

In this first case, as illustrated in Fig. 4, spacecraft potentials settle to steady values after a transitory regime, here lasting $0.4 \mathrm{~s}$, due to the instantaneous Sun lightning of the satellite inherent to simulation start. Steady potentials reached by satellite elements are displayed on Tab. 2.

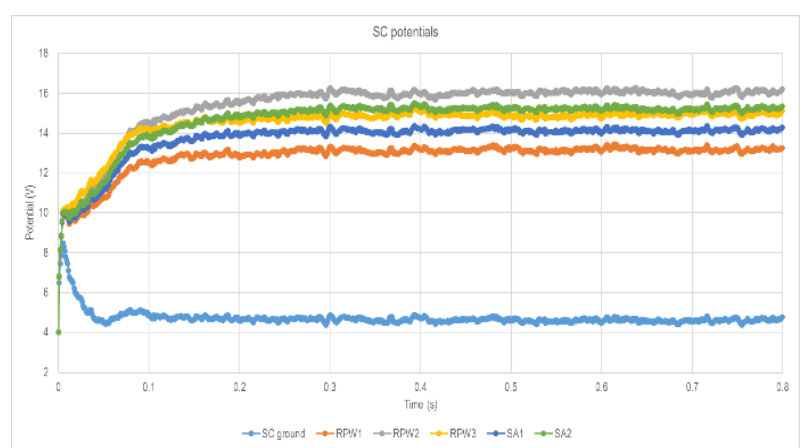

Figure 4. Spacecraft potentials versus time (light blue: spacecraft ground, orange: $R P W 1$, grey: $R P W 2$, yellow: $R P W 3$, dark blue: SA1, green: $S A 2$ )

These are average values calculated between $t=0.4$ and $t=0.8 \mathrm{~s}$. Standard deviations of those values during this period are also indicated as a proof of numerical stability. The two solar panels sunlit faces are identified as SA1 (on the $-X$ side of the simulation box) and SA2 on the opposite side. The three antennas are identified as RPW1 along the $Y$ axis, RPW2 towards $-X$ and RPW3 on the $+X$ side of the simulation box.

\begin{tabular}{|c|c|c|}
\hline $\begin{array}{c}\text { In average after } \\
\boldsymbol{t}=\mathbf{0 . 4} \mathbf{~ s}\end{array}$ & Potential $(\mathbf{V})$ & $\begin{array}{c}\text { Standard } \\
\text { deviation }(\mathbf{V})\end{array}$ \\
\hline SC ground & 4.61 & 0.08 \\
\hline RPW1 $(+Y)$ & 13.17 & 0.09 \\
\hline RPW2 $(-X)$ & 16.04 & 0.11 \\
\hline RPW3 $(+X)$ & 14.93 & 0.10 \\
\hline SA 1 $(-X)$ & 14.13 & 0.09 \\
\hline SA 2 $(+X)$ & 15.23 & 0.08 \\
\hline
\end{tabular}

Table 2. Average steady potentials on Solar Orbiter elements

Fig. 5 shows the plasma potential in the $X-Z$ plane cutting through the middle of the sunshield and solar panels (i.e. $Y=0$ ). Negative potential areas appear ahead of the spacecraft (because of high densities of secondary and photoelectrons) and downstream of it as a consequence of the ion wake (protons' mean flow is along $-Z$ ) and high densities of secondary electrons. Maximum potential of $15.2 \mathrm{~V}$ is on the front face of the $-X$ sided solar array. Downstream of the satellite body the tip of the rear boom crosses the $Y=0$ plane which is the reason for the small positive potential bump in that region. Fig. 6 displays the plasma potential in the plane containing the three RPW antennas. This particular cut through the simulation also shows the influence of the 


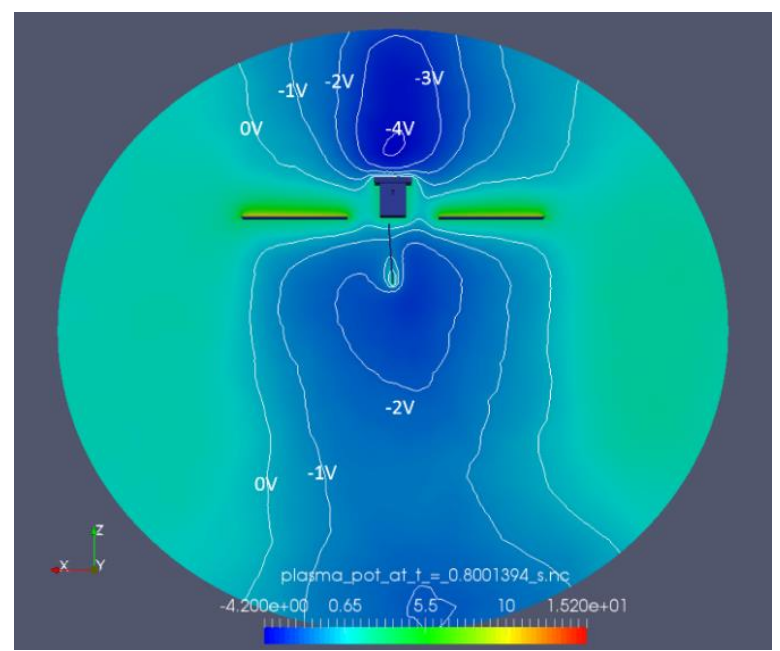

Figure 5. Plasma potential (V) in the $X-Z$ plane going through the middle of sunshield and solar panels

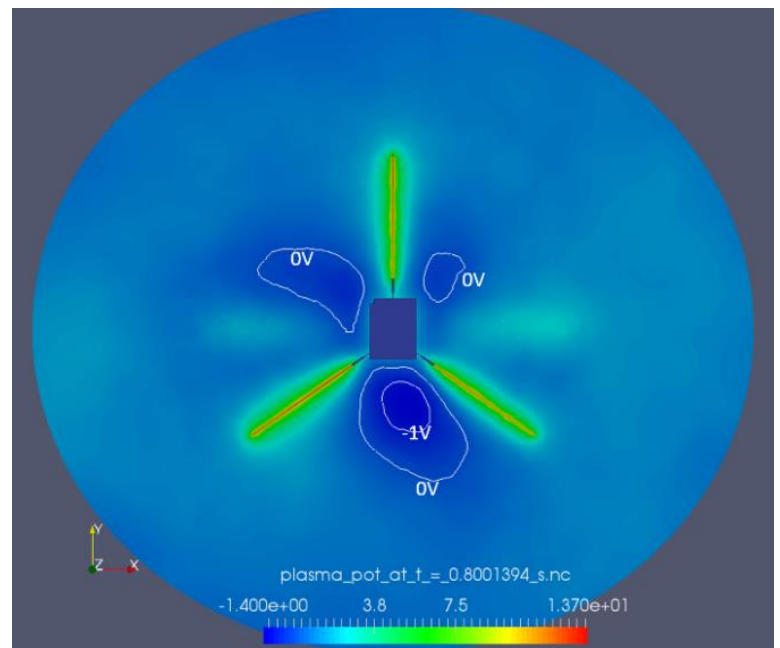

Figure 6. Plasma potential (V) in the plane of the RPW antennas (the $X-Y$ plane)

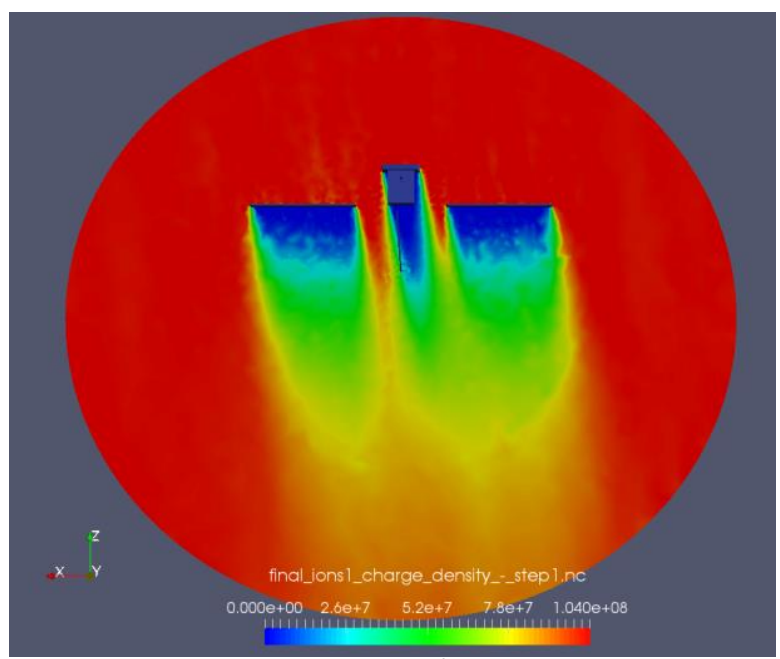

Figure 7. Ion density $\left(\mathrm{m}^{-3}\right)$ in the $X-Z$ plane

solar array cover glasses in the vicinity of the stacers. We emphasize that Figures 5 and 6 show the potential in the plasma frame, not in the spacecraft frame (the plasma flows with respect to the spacecraft). Fig. 7 is the ion density map in the $X-Z$ plane, clearly showing the ion depletion due to the combination of the proton bulk velocity (the solar wind velocity) towards $-Z$ and the spacecraft orbital velocity towards the $+X$ direction. A more in detail discussion of the wake formation can be found in [4].

Let us now estimate the effective length for the three couples of RPW antennas. The effective length $\boldsymbol{L e f f}_{A B}$

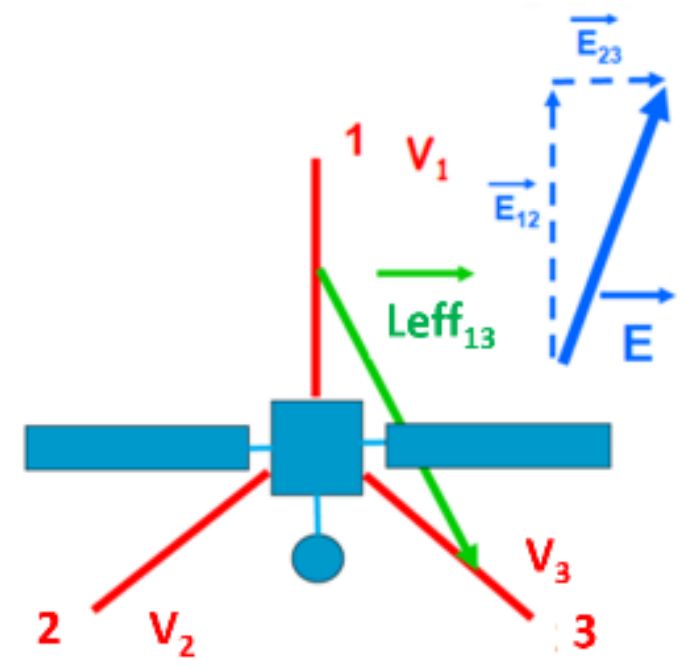

between any two antennas A and B is defined by assuming a linear relation between the external electric field $\boldsymbol{E}$ and the potential difference $\partial \Phi_{A B}$ between $\mathrm{A}$ and B: $\partial \Phi_{A B}=\boldsymbol{L e f f}_{A B} \boldsymbol{E}$ (see the sketch above). In the simulation the ambient field $\boldsymbol{E}$ is user defined while the potential differences $\partial \Phi_{A B}$ for the three antenna couples are computed by the simulation (see Table 3). Arbitrarily assuming that the directions of the effective length vectors $\boldsymbol{L e f f}_{\boldsymbol{A} \boldsymbol{B}}$ are aligned with the sides of a triangle connecting the antenna centers (see Fig. 8) an effective length can be given for each couple of antennas.

\begin{tabular}{|c|c|c|}
\hline $\begin{array}{c}\text { In average after } \\
\boldsymbol{t}=\mathbf{0 . 4} \mathbf{~ s}\end{array}$ & Potential $\mathbf{( V )}$ & $\begin{array}{c}\text { Standard } \\
\text { deviation }(\mathbf{V})\end{array}$ \\
\hline$\Delta \Phi_{1-2}=\Phi 1-\Phi 2$ & -2.87 & 0.07 \\
\hline$\Delta \Phi_{1-3}=\Phi 1-\Phi 3$ & -1.76 & 0.08 \\
\hline$\Delta \Phi_{2-3}=\Phi 2-\Phi 3$ & 1.11 & 0.11 \\
\hline
\end{tabular}

Table 3. Average differences of potential on $R P W$ antennas

For symmetry reasons, given that $\boldsymbol{E}$ is along $Y$ axis, Leff $_{12}$ and Leff $_{13}$ should be identical but the potential difference between 1 and 2 being stronger than between 1 and 3 gives Leff $_{12}=13.66 \mathrm{~m}$ and Leff $_{13}=8.38 \mathrm{~m}$. 


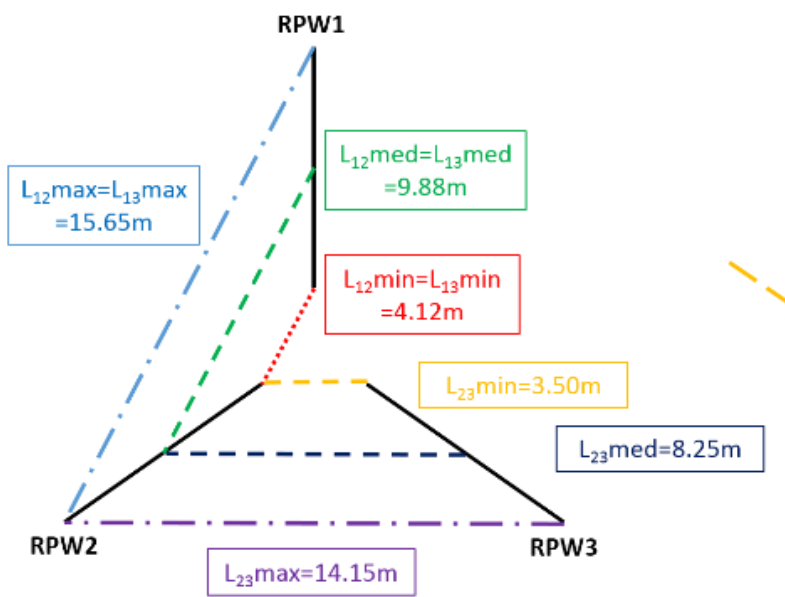

Figure 8. RPW configuration and effective lengths

Fig. 8 shows that the obtained average effective length of $11 \mathrm{~m}$ is in the range medium to maximum separation distance between the corresponding antenna couples. Where does this 5 meters discrepancy between Leff $_{12}$ and Leff $_{13}$ comes from? The answer is the strong magnetic field which forces the electrons to be magnetized (the field in the simulation is intentionally much stronger than in the real wind in order to emphasize a strong electric field on the RPW antennas). Indeed, in the spacecraft frame magnetized particles (with a Larmor radius smaller than the simulation domain) are subject to a drift motion given by:

$$
V_{d}=\left(\boldsymbol{E}_{\text {perp }} \times \boldsymbol{B}\right) / B^{2}
$$

In our case the drift motion is towards the $-X$ direction. On the other hand the Larmor radius of a charged particle of mass $m$, charge $q$ and velocity $v \_$perp component perpendicular to the magnetic field is given by:

$$
r=m v \_p e r p /(q B)
$$

For secondary and photoelectrons $v_{d} \sim-6 \times 10^{4} \mathrm{~m} / \mathrm{s}$ in the $-X$ direction (opposite to $V_{S C}$ but of the same order) and $r \sim 1 \mathrm{~m}$. The resulting spatially asymmetric photoelectron distribution is clearly visible in Fig. 9.

Secondary electrons due to electron and proton impacts behave similarly as shown in Fig. 10 where it appears that RPW2 and 3 do not reach the same potential, because of the non-symmetric flow of low energy electrons in the space between the two antennas.

This is the direct effect of using $\boldsymbol{B}$ and $\boldsymbol{V}$ to generate the convection electric field. In reality the magnetic field wouldn't be so strong and such perturbations shouldn't appear on RPW antennas.

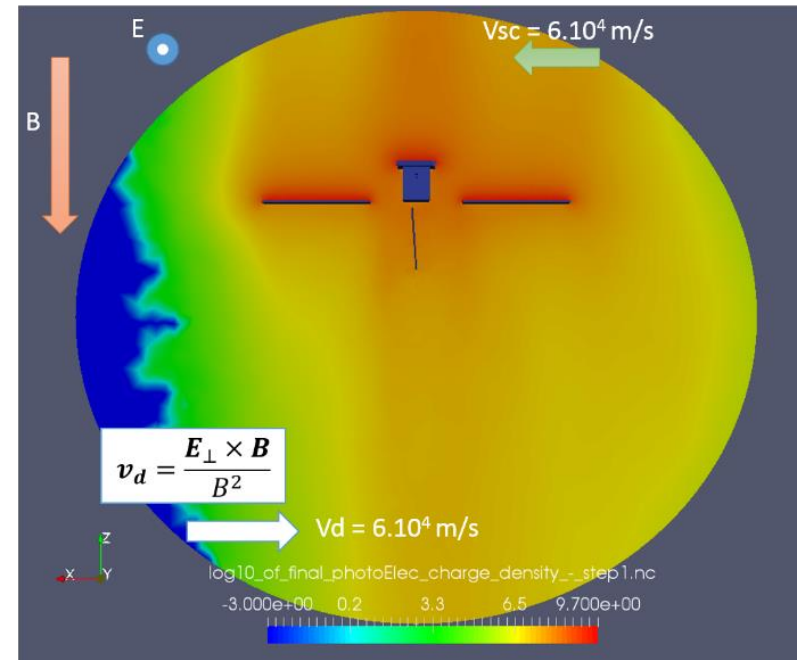

Figure 9. Log of photoelectron density in the X-Z plane

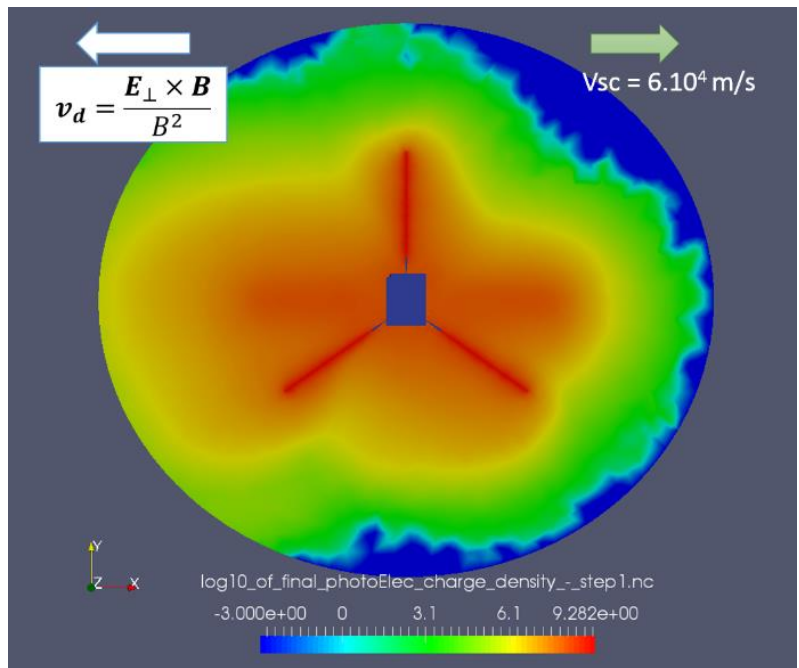

Figure 10. Log of photoelectron density in the X-Y plane

\subsection{Conclusion for RPW antennas}

The PIC simulations presented above can help to understand RPW measurements at perihelion. Using a magnetic field intensity higher than the expected ambient value provided a clear insight into the physical mechanisms which affect the potentials on the antennas. The specific case with particle drift velocity direction normal to the RPW plane has already been performed and will be presented in the next future. We note that the substantially smaller magnetic field in the real solar wind may allow a more universal determination (independent of magnetic field orientation) of the antennas' effective lengths. On the other hand, smaller ambient electric fields will be more easily overwhelmed by the local (spacecraft induced) fields and make the effective lengths become dependent on the actual plasma parameters. 


\section{THE EAS INSTRUMENT IN THE SIMULATION}

In the following a simulation of the Electron Analyzer System measurement at perihelion will be presented. This case was previously investigated [1], without taking into account the magnetic field. The spacecraft geometry used at this time is now outdated but globally respects main element dimensions and configurations. In the near future EAS numerical model will be implemented into the case presented in Section 3. But the following case already presents new SPIS capabilities of modelling numerical particle detectors and what type of outputs we want to obtain.

\subsection{Numerical EAS modelling and simulation configuration}

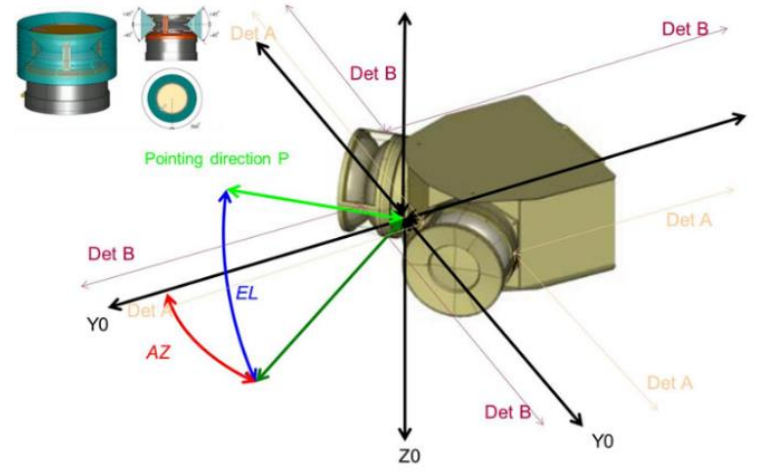

Figure 11. EAS CAD model and configuration

EAS technical properties have already been introduced in Section 1. Its design is displayed on Fig. 11 and GMSH model of the instrument used in SPIS on Fig. 12. For this type of detector, with particle entrance for each EAS sensor as a circular ring, it is necessary to consider each EAS entrance as the sum of eight curved surfaces, in order to limit the curvature of each surface detector. Indeed, each surface is considered in SPIS model as a particle detector with a unique local detector basis $\left(X_{D}\right.$, $Y_{D}, Z_{D}$ ) defined so as the $Z_{D}$ axis is pointing into the detector, normal to the surface. This definition allows defining properly the acceptance angles for incoming particles in this basis. This is why each particle detector has to remain relatively "flat". The EAS instrument is thus composed in this model of 16 particle detector surfaces, each one providing its own outputs which will have to be combined for a global overview of EAS results. For all cases presented here the acceptance angles are $\pm 90^{\circ}$ in azimuth $(A Z)$ and $\pm 45^{\circ}$ in elevation $(E L)$. This way the entire field of view of both detectors covers the $4 \pi$ sr of the environment, as in reality.

Plasma parameters are identical to the ones presented on Table 1. Environment parameters used in SPIS, except that we do not consider any magnetic field or a spacecraft velocity in $X$ direction. Simulation configuration is the same that was detailed in Section 2.2. EAS outputs are presented hereafter.

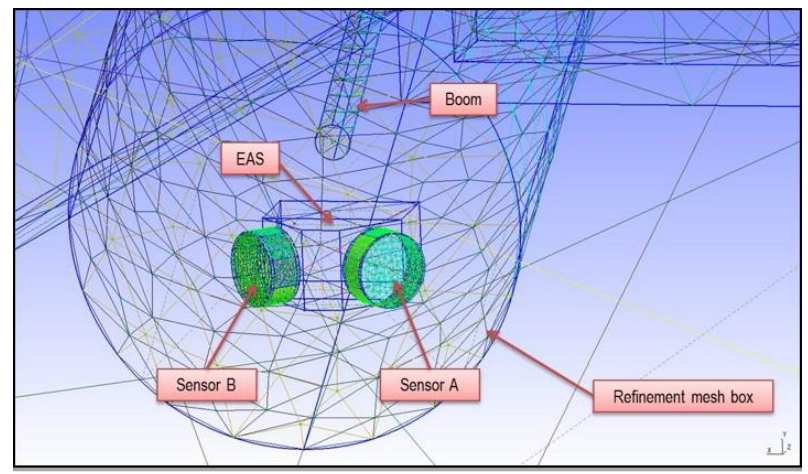

Figure 12. GMSH model for EAS used in SPIS

\subsection{EAS results}

For this case without $\boldsymbol{B}$, this spacecraft geometry and covering materials, all conducting Solar Orbiter surfaces (including EAS) are set at $+3.1 \mathrm{~V}$. Front faces of solar arrays (dielectric) are charged at $+14.1 \mathrm{~V}$, and the High Gain Antenna (HGA, dielectric at this time), is charged at a potential of $+9 \mathrm{~V}$.

We can further investigate the global plasma behaviour around our object. For instance, the plasma potential in the $X-Z$ plane (Fig. 13) shows that we recover the same electrostatic phenomena in the ram (high densities of secondary and photoelectrons, as it appears on Fig. 14) and the in wake (lack of protons and high density of secondary particles). Contrary to the previous RPW case including $\boldsymbol{V} \times \boldsymbol{B}$ effect: no drift or expansion of low energy electrons appears.

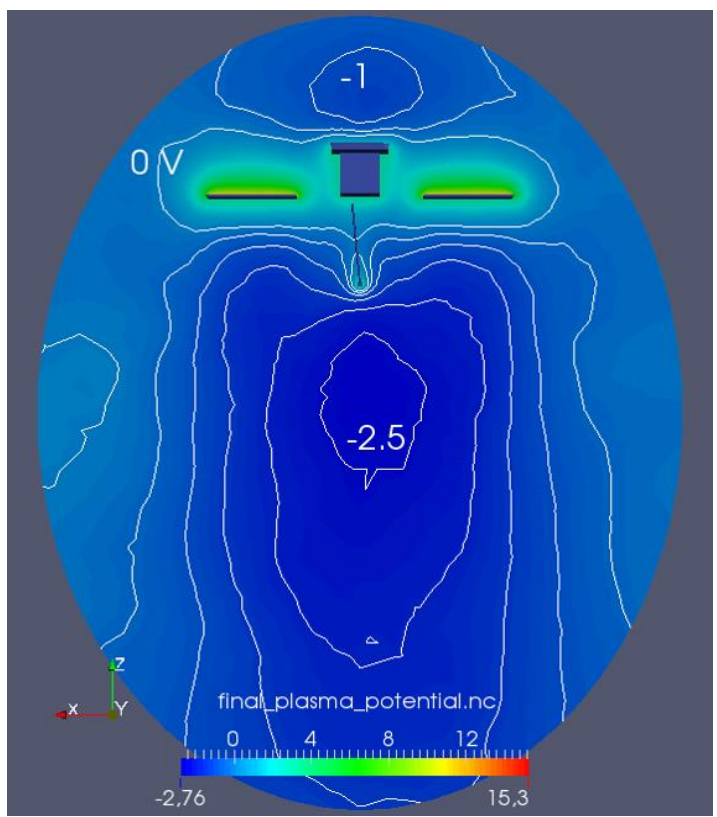

Figure 13. Plasma potential around Solar Orbiter in the $X-Z$ plane 


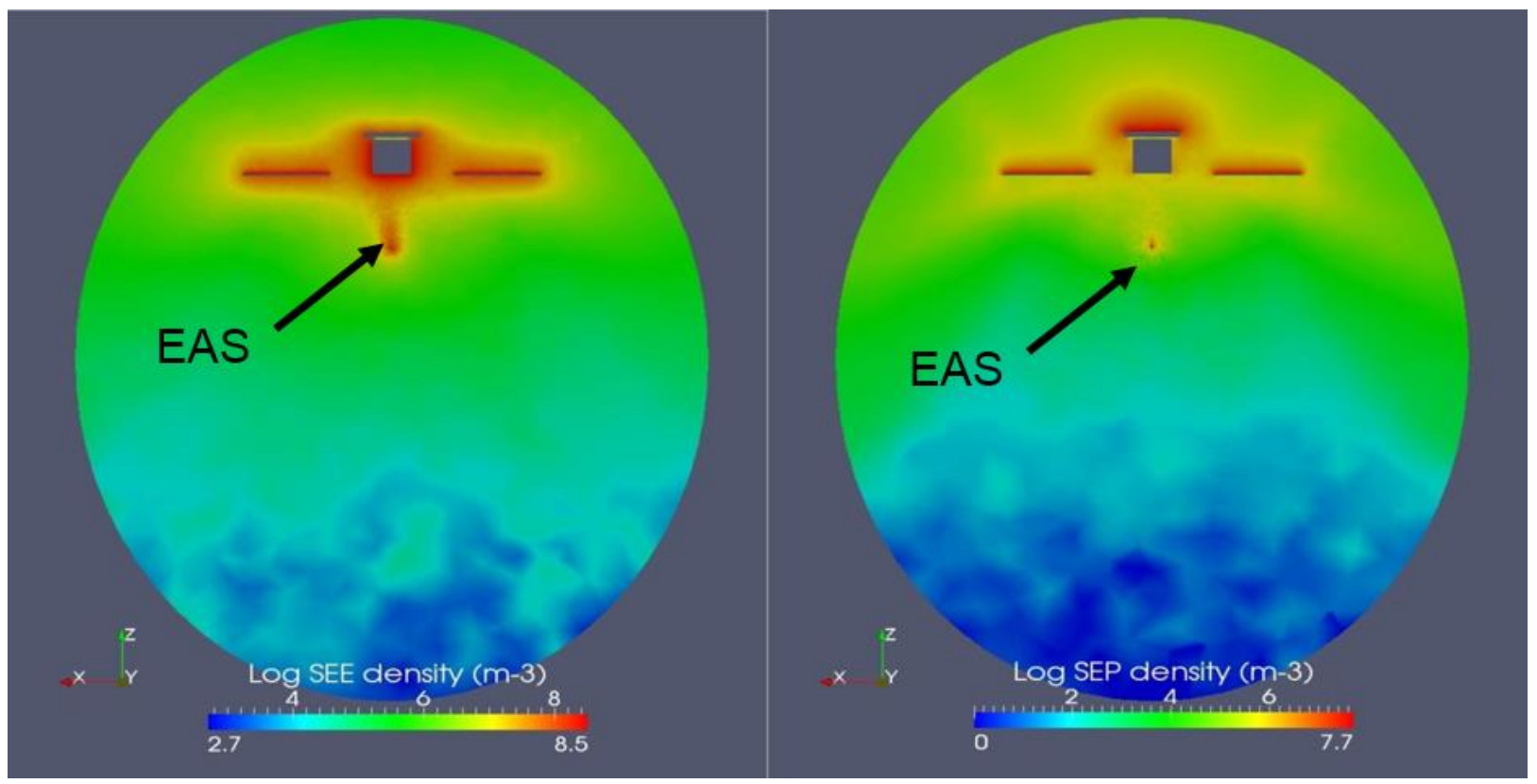

Figure 14. Log of secondary electron (left) and photoelectron (right) density in the X-Z plan

Then, what does EAS measure? First let's have a look on the Energy Distribution Function (EDF) of thermal electrons (see Fig. 15). The environment yellow curve is the electron distribution from environment as described in Table 1. Environment parameters used in SPIS, and the one that EAS should measure if there were no plasma disturbances due to spacecraft/plasma interactions.

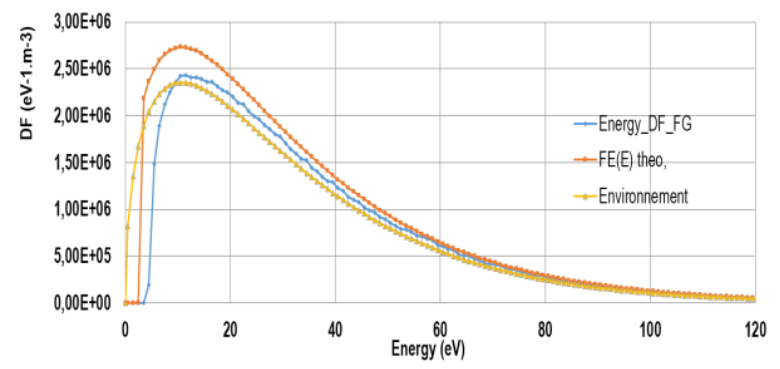

Figure 15. Energy distribution functions of thermal electrons on EAS

The orange curve $F E(E)$, represents the environment that should be theoretically measured by EAS if there was only this instrument charged at its potential of $+3.1 \mathrm{~V}$ in the simulation box, with no spacecraft, no wake nor any potential barrier around EAS. It is calculated analytically using Liouville's theorem [3]. This distribution function is thus based on the true environment, but shifted of $3.1 \mathrm{eV}$ (indeed no electrons can reach the detector with a potential energy inferior to EAS potential), and increased after $3.1 \mathrm{eV}$ because of the attracting electric potential of EAS.

Finally, the blue curve Energy DF FG represents the simulated measurement of thermal electrons. The measurement results in a combination between the true environment and the theoretical measure of EAS alone. Indeed, the analytical modelling using Liouville's theorem assuming all possible trajectories are filled regarding the expected distribution function in a local non zero potential is only valid for a single detector immersed in the plasma. As here the entire Solar Orbiter structure is positively charged (at values that can reach $+14.1 \mathrm{~V}$ on the Solar panels), many thermal electrons are attracted in those areas and fewer on EAS detectors which results in a lack of information on the low energy electrons in the simulated measures. It can be seen on the difference of amplitude between the maximums of the blue and orange curves.

The corresponding densities calculated through integration of thermal electrons $E D F$ (Fig. 15) are:

- Undisturbed environment (yellow): $N_{0}=$ $1.04 \times 10^{8} \mathrm{~m}^{-3}$. Same values are found as this model is practically not truncated (the highest temperature considered is $200 \mathrm{eV}$ i.e. almost 10 times the thermal electrons temperature modelled),

- Theory (orange): $N_{\text {theo }}=1.16 \times 10^{8} \mathrm{~m}^{-3}>N_{0}$ because of EAS potential $(+3.1 \mathrm{~V})$,

- Measurements (blue): $N_{\text {meas }}=1.01 \times 10^{8} \mathrm{~m}^{-3}<$ $N_{\text {theo }}$ because of the spacecraft charged structure and the plasma disturbances (ion wake, solar panels, HGA, potential barriers).

It can be noticed that the simulated electron measurements (blue curve) is lower than the theoretical red curve (analytical model): it sounds like low energy electrons (visibly between 3 and $5 \mathrm{eV}$ ) are missing. The explanation is that the EAS instrument is surrounded by negative potential barriers between -1.5 and $-2.5 \mathrm{~V}$ : they 
filter the low energy electrons. The particles which cross those barriers are then accelerated by EAS potential. The minimum energy of those elements becomes: $1.5+$ $3.1=4.6 \mathrm{eV}$. Negative potentials around the particle detector increase the SEEE densities through electron impacts. $E D F$ reaches $5 \times 10^{7} \mathrm{~m}^{-3} . \mathrm{eV}^{-1}$ for SEEE and $2.5 \times 10^{6} \mathrm{~m}^{-3} \cdot \mathrm{eV}^{-1}$ for the primary electrons (factor 20 ).

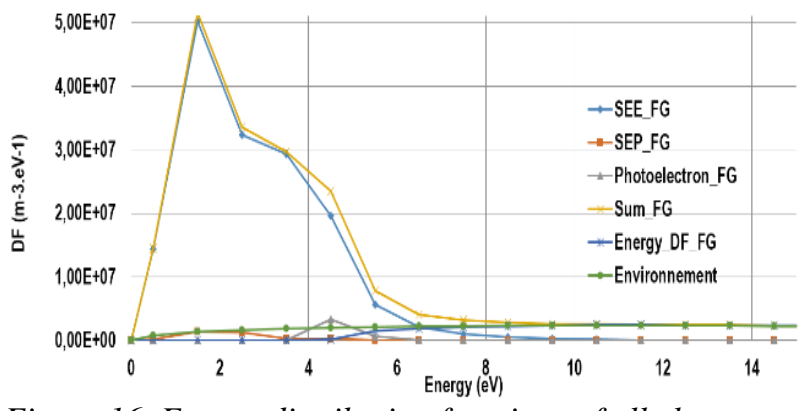

Figure 16. Energy distribution functions of all electrons on EAS

The simulated measurements of thermal electrons give a discrepancy of $2.8 \%$ regarding $N_{0}$. Adding to the measurements photoelectrons, SEEE and SEEP (Fig. 16) gives a total density of measured electrons (the purple Sum curve) of $N_{\text {total }}=2.42 \times 10^{8} \mathrm{~m}^{-3}$ (a difference of $132 \%$ regarding $N_{0}$ ). The low energy ranges are highly dominated by SEEE.

Other results produced with post processing the particle detector outputs are the 2D maps of detection of thermal electrons regarding EAS pointing direction (Fig. 17).

They can provide information regarding the instrument field of view and help answer questions such as: where are the physical or electrostatic obstacles to electron detection and what are the consecutive impacts on measurements, from which directions come the highest/lowest particle fluxes, etc. The EAS detailed field of view, with definition of pointing angles azimuth (AZ) and elevation (EL), was presented on Fig. 11. The limits of $\mathrm{AZ}$ acceptance when $\mathrm{EL}=0^{\circ}$ are also represented (with thin purple and orange arrows) to show that both sensors are needed to cover the entire field of view. Fig. 17 is the counting map of thermal electrons detected by the entire EAS instrument, regarding the pointing direction (AZ and EL angles). In order to compute this map, it was necessary to create bins in $\mathrm{AZ}$ and $\mathrm{EL}\left(10^{\circ}\right.$ bins in this case) constituting the angular resolution (and similar to the real EAS angular resolution). Only electrons with less than $15 \mathrm{eV}$ energy have been treated (as low energy electrons are the most disturbed by the spacecraft presence in the environment).

Note that other types of resolution/energy ranges can be of course selected to better fit other requirements. In red are indicated the objects pointed with the corresponding values of $\mathrm{AZ}$ and EL: the Solar Arrays (SA) and the spacecraft body (SC) when looking behind EAS (note that the objects positions are quite approximated). The bin colors (from blue to red) indicate the number of electrons counted in each bin direction. In the same Fig. 17 the effect of the physical body clearly blocking particle arrival is manifest. The Solar panels do not visibly affect detection in their specific direction as they are quite thin regarding the bin precision and close to the spacecraft body. At $\mathrm{AZ} \sim 90^{\circ}$ and $\sim 270^{\circ}$ (and EL $0^{\circ}$ ) there is a lower particle detection due to several causes. First the pointing direction skimming or targeting the charged detectors themselves. Indeed, it appears in Fig. 11 that at $\mathrm{AZ} \sim 90^{\circ}$ and a null elevation, detector $\mathrm{B}$ on the left points towards the detector $\mathrm{A}$ on the right, charged at $+3 \mathrm{~V}$. Secondly the connection between the two detectors fields of view: indeed, at these specific directions one detector begin its acceptance domain while the other ends it, see Fig. 11, which also explains the loss of particles when both detectors point the null AZ and EL direction. When EL $\sim 0^{\circ}$ the sensors have no common pointing directions. On the contrary the enhancement of electron detection at high EL values is simply due to a geometric factor: when EL approaches $\pm 90^{\circ}$, both sensors have common pointing direction, whatever the $\mathrm{AZ}$ value considered. It results in an increase in particle counting. The HGA and the two lower RPW antennas have also a blocking effect on electrons $\left(\mathrm{EL} \sim-45^{\circ}, \mathrm{AZ} \sim 180^{\circ}\right.$ ), this is why EAS collects fewer particles when pointing towards EL $90^{\circ}$, rather than $\mathrm{EL} \sim+90^{\circ}$.

\subsection{Conclusion for numerical EAS measurements}

This Section also showed (as for RPW antennas in previous Section) how new SPIS capabilities allow to handle numerical instrument measurements and understand those results: here concerning origin of parasitic particle detection, computation of biases, etc. Even though this case has been performed using an outdating model of Solar Orbiter, neglecting electromagnetic fields that will definitely exist at satellite perihelion, it gives an explicit example of what our work will focus on in the next future.

\section{CONCLUSION}

A preliminary study of Solar Orbiter and its scientific instruments, RPW and SWA-EAS, behaviour in the solar wind at perihelion (0.28 AU from the Sun) was presented. Simulations show various non negligible effects that should be considered in order to understand and correctly interpret the plasma and field measurements: surface charging and electrostatic sheath, secondary and photoelectron emission and collection, $\quad \boldsymbol{V} \times \boldsymbol{B}$ induced electric field and corresponding drift velocity (and gyration) of low energy particles, etc. Combined effects of these phenomena will definitely affect and complicate surrounding environment analysis. As demonstrated in 


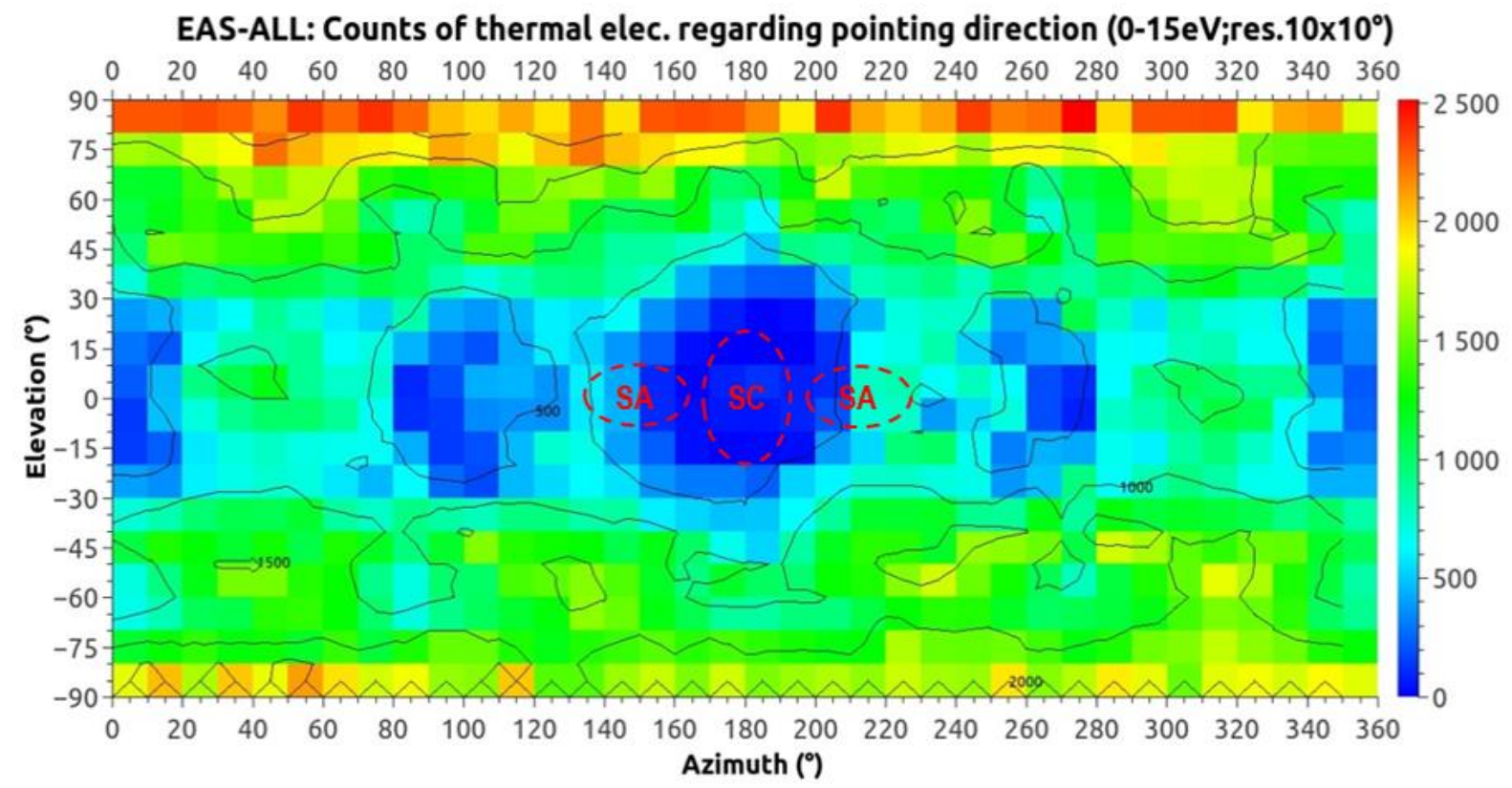

Figure 17. Counting map of thermal electrons detected by EAS

this study, numerical simulations help to separate various interactions and to distinguish their effects on obtained data.

Future work is foreseen to include recent updates of Solar Orbiter surface material properties, and to take into account smaller values of the magnetic field and its proper orientation at the satellite perihelion. Independent currents will also be injected into RPW stacers in order to lower down their electrostatic potentials and get closer to plasma potential.

\section{REFERENCES}

1. Guillemant, S. Study and Simulations of Spacecraft/Plasma Interaction Phenomena and their Effects on Low Energy Plasma Measurements. Earth and Planetary Astrophysics [astro-ph.EP]. Université Paul Sabatier Toulouse III, 2014.

2. Roussel, J.-F., Rogier, F., Dufour, G., Matéo-Vélez, J.-C., Forest, J., Hilgers, A., Rodgers, D., Girard, L. and Payan, D. SPIS Open Source Code: Methods, Capabilities, Achievements and Prospects. IEEE Trans. Plasma Sci., 36, 23602368, doi:10.1109/TPS.2008.2002327, 2008.

3. Hilgers, A., S. Clucas, B. Thiébault, J.-F.s Roussel, J.-C. Matéo-Vélez, J. Forest, and D. Rodgers, Modeling of Plasma Probe Interactions With a PIC Code Using an Unstructured Mesh, , IEEE Trans. Plasma Sci, Vol 36, No5 Part2, pp 23192323, DOI: 10.1109/TPS.2008.2003360, Oct 2008.

4. Guillemant, S., Génot, V., Matéo-Vélez, J.-C., Sarrailh, P., Hilgers, A. and Louarn, P. Simulation
Study of Spacecraft Electrostatic Sheath Changes With the Heliocentric Distances from 0.044 to 1 AU. Plasma Science, IEEE Transactions on, Volume: PP, Issue: 99, 2013. 